\title{
Fault Diagnosis Based on Multi-sensor Data Fusion for Numerical Control Machine
}

\author{
http://dx.doi.org/10.3991/ijoe.v12i02.5040 \\ WEN Yan, TAN Ji-wen, ZHAN Hong, SUN Xian-bin \\ Qingdao Technological University, Qingdao, China
}

\begin{abstract}
Fault diagnosis for numerical control machine is more difficult than that for other mechanical equipments due to its structural complexity and the coupling feature among different faults. In order to improve the accuracy and reliability of fault diagnosis for numerical control machine, an intelligent fault diagnosis model is studied. Besides the traditional method that multiple sensors are mounted on different locations, internal operation parameters from machine tool itself or NC program are introduced into the condition monitoring system because numerical machine tool is equipped with different kinds of sensors. These two information sources establish the multi-dimensional information system which provides the original information for diagnosis. On this base, the method based on multi-sensor data fusion is developed in this paper. Multiple characteristic parameters in time domain, frequency domain and timefrequency domain are extracted from the processed signal to mine the fault information. The sensitive parameter set which is regarded as the input characteristic vectors of classifiers is obtained on the base of correlation analysis. Multiple classifiers are enabled respectively and simultaneously to fuse all the sensitive parameters quantitatively and diagnose the fault type. Finally the results of multiple classifiers are fused in the form of global decision fusion by the method of fuzzy comprehensive evaluation to obtain the final diagnosis result. The determination method of weight based on classifier output's entropy is discussed in this paper and the formula is given. This model and method has been tested in rolling bearing fault diagnosis for numerical control machine and the results of the proposed model show which is effective and versatile.
\end{abstract}

Index Terms - multi-sensor, data fusion, numerical control machine, hybrid intelligent model, fault diagnosis

\section{INTRODUCTION}

The numerical control machines which have been widely used are the foundation of equipment manufacturing industry. Since the numerical control devices are under the running condition such as high-speed and variable load, frequent reversing, vibration and impact, friction and wear and etc., the typical parts' performance may occur variance to influence processing quality and precision, which even lead to the equipment failure. So the condition monitoring and fault

diagnosis research for the numerical control devices is crucial to preventive and predictive maintenance, proper functioning of device and machining quality of work pieces. Most self-diagnosis system of numerical control machine [1-[2] can not diagnose faults in mechanical parts, so the question of fault diagnosis for mechanical parts has been attracting much attention.
In order to improve the accuracy of diagnosis, there are two scopes which are focus on by researchers: advanced signal processing methods and artificial intelligence technology. In recent years the processing methods of nonstationary signal such as wavelet analysis \& wavelet pocket analysis, Empirical Mode Decomposition (EMD), stochastic resonance, etc. are used in fault diagnosis to mine more effective fault information. The technology of artificial intelligent including expert system, neural network, genetic algorithm and fuzzy logic, etc. is applied in fault diagnosis system to improve the degree of intelligent diagnosis. These methods and techniques are all applied in the diagnosis system which acquire better result in practice [3]. With the application and development of these new methods and techniques, the question that these methods have their own advantages and disadvantages and only can be applied in particular conditions is emerged [6]. So the method of hybrid intelligent fault diagnosis has been studied widely [7]. In these researches, multiple advanced signal processing method and artificial intelligent techniques are utilized simultaneously. But in most diagnosis system only one kind of information (such as vibration information) is used which often cause the incompleteness of information about objects especially in condition of fault diagnosis for complicated system. The lack of information even leads to misdiagnosis. The second point is that the extracted character features are concentrated in a certain single domain. The character features of other domains are abandoned. In fact the information of fault is reflected in time domain, frequency domain and time-frequency domain only in different degree.

Aiming at the problem existing in hybrid intelligent fault diagnosis, the diagnosis structure model based on multi-dimensional information system and multi-level information fusion is established, as shown in figure 1. The multi-dimensional information system providing the original information for fault diagnosis has two independent information sources: external sensors and internal running parameters. External sensors including vibration sensors, temperature sensors noise sensors, visual sensors and so on which are mounted at different locations. The type of sensor can be chosen according to the characteristics of fault. Because numerical control machine itself is equipped with many high accuracy sensors to ensure the accuracy of processing. So the running status parameters from numerical control machine or NC program form the internal sources of information. The multi-dimensional information system provides complete information to diagnosis system. The signals from different sources are investigated in the time domain, frequency domain and time-frequency domain respectively. The characteristic 
features are extracted in every domain. In order to choose sensitive fault characteristic features and simplify the structure of classifier, the select method of characteristic features based on correlation analysis is studied. Feature fusion is completed in this stage. In the stage of pattern recognition, Two-level diagnosis network mode is adopted. The first level network is parallel structure which composes of multiple classifiers. These classifiers analysis sensitive characteristic features independently and diagnose fault type. The results of the classifiers in the first level are sent to the second level network for the deeper integration by fuzzy comprehensive evaluation. The method of classifiers weighted based on diagnostic accuracy is researched. The final diagnosis conclusion is gained after two levels fusion.

In this fault diagnosis model many measurements are taken to reduce the uncertainty in the diagnosis. Firstly the increase of numerical control machine's running parameters information source forms multi-dimensional information system which can reflect the fault information comprehensively and completely. Secondly the multilevel fusion mines the effective fault information hidden in the original signals. Thirdly the classifiers' results are fused based on fuzzy comprehensive evaluation which is the simulation of the method of human decision. Every classifier's result is defined as a factor in diagnosis decision. Various intelligent fault diagnosis technologies are combined together in this model.

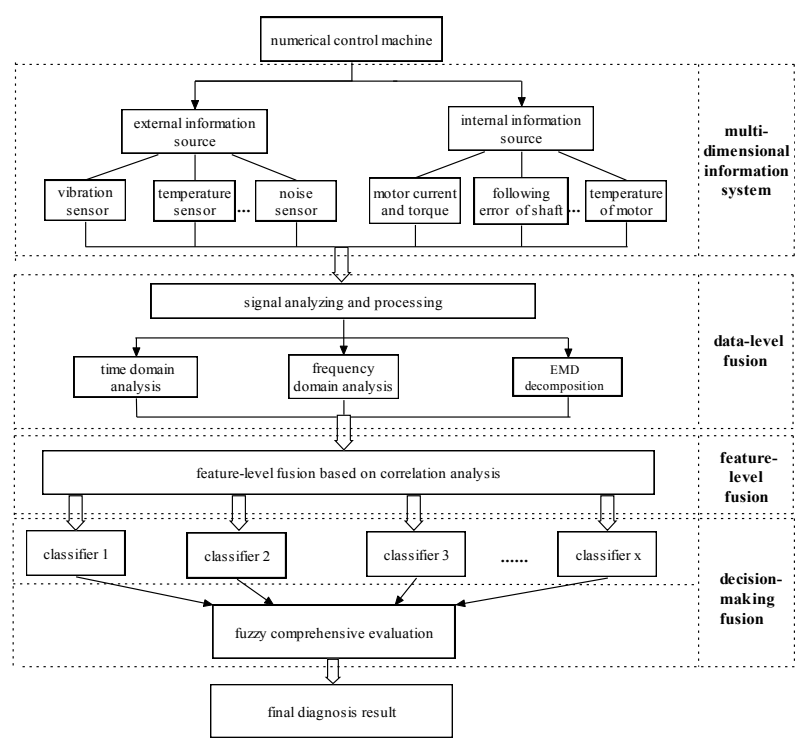

Figure 1. Fault diagnosis model

\section{MUlTi-DiMENSIONAL INFORMATION SYSTEM}

Information is absolutely necessary for fault diagnosis. Correct diagnosis conclusion is likely to gain only in the status that the running state of numerical control machine can grasp timely and comprehensively. Due to the complexity coupling between subsystems of numerical control machine, the traditional fault diagnosis way of single sensor is tend to cause the uncertainty of information, which may lead to a misdiagnosis or missed diagnosis conclusion. Thus multi-source information fusion technique is introduced into the fault diagnosis for numerical control machine. Many sensors which are various types such as vibration, noise, temperature, cutting force and image etc. [9] form the sensor array. The technology of multi-sensor fusion brings a new opportunity for fault diagnosis of numerical control machine. However these sensors are additional structure against machine tools. Installation of these sensors is inconvenient due to the limitation of machine tool structure or size. On the other hand, the signals become very weak because of the propagation medium's number and long path length. The two points are the disadvantages of multiple external sensors method.

Numerical control machine itself is equipped with many high-precision sensors such as grating, rotary encoder, current sensor, temperature sensor to guarantee the accuracy. And closed loop or half closed loop control method is often adopted. That is to say, the operation status of the numerical control machine can be gained in real time by many parameters such as the current or torque, temperature of motor and different kinds of errors. With the appearance and development of open numerical control machine tools in recent years, the internal sensors' signals of numerical control machine can be achieved directly under the online state [10]. The information gathered from internal sensors is very useful for fault diagnosis. This method doesn't need any additional sensor or device, and the signals are more reliable compares with external sensor method.

So in the diagnosis model proposed in this paper external sensors and internal information of numerical control machine tool are the two information sources on condition monitor and fault diagnosis system. The problems of poor ability to reflect the fault information when the built-in sensor is far away from the fault [11] and the limit of installation position of external sensor are solved through the combination of information. Different kinds of multiple sensors mounted on different locations and multiple parameters gained from numerical control machine tool provide complementary information to characterize the state of the machine tool.

\section{SignAl ANALYZING AND PROCESSING}

Because the different type, degree or location of faults, the fault information will reflect in the time domain, frequency domain and time-frequency domain only in different levels. The necessary analysis and processing of the original signal is needed to find the effective information.

Time-frequency analysis is a powerful tool for analyzing time-varying, non-stationary signals, and can describe the relationship between signal frequency changes over time. The method Empirical Mode Decomposition [13] is used commonly in signal time-frequency analysis.

The basic idea of EMD is to decompose a signal into the sum of several multi-scale signals, called the intrinsic mode function (IMF) [14]. The original signal is decomposed into multiple IMFs and one residual component, as Eq.(1).

$$
x(t)=\sum_{i=1}^{n} c_{i}(t)+r_{n}(t)
$$

In the field of signal and test technology, the energy of signal is as Eq.(2).

$$
E(i)=\int_{-\infty}^{+\infty}|x(t)|^{2} d t
$$


when the signal $\mathrm{x}(\mathrm{t})$ is a discrete signal, the total energy is the sum of every interval energy.

$$
E(i)=\sum_{t=0}^{n}\left|x^{2}(t)\right| \Delta t
$$

Where $\Delta t$ is sample interval.

When the fault occurs, the frequency of vibration changes obviously. So the energy value of same frequency band is different which cause the different energy distribution of signal, as shown in fig 2. That is to say, the energy of every frequency band contains abundant information about fault. The signal can be decomposed into some frequency bands automatically by method of EMD. When the fault occors, the energy of IMFs also change, which are treated as characteristic feature.

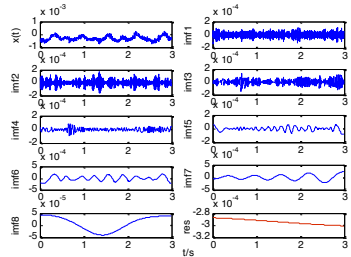

(a)normal

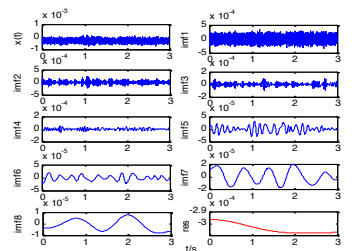

(b)inner ring fault
Figure 2. EMD decomposition in different faults

So in order to find the effective fault information, characteristic parameters of each domain are extracted. These parameters constitute the original failure data set which is listed in table I.

TABLE I.

CHARACTERISTIC PARAMETERS

\begin{tabular}{|l|l|c|}
\hline $\begin{array}{c}\text { Mode of signal } \\
\text { processing }\end{array}$ & \multicolumn{1}{|c|}{$\begin{array}{c}\text { Names of characteristic } \\
\text { parameters }\end{array}$} & $\begin{array}{c}\text { Number of charac- } \\
\text { teristic parameters }\end{array}$ \\
\hline time domain & $\begin{array}{l}\text { peak, RMS value, average } \\
\text { root, average mean, kurtosis, } \\
\text { skewness, waveform factor, } \\
\text { pulse factor, peak factor, } \\
\text { margin factor, kurtosis factor }\end{array}$ & 11 \\
\hline $\begin{array}{l}\text { frequency do- } \\
\text { main }\end{array}$ & $\begin{array}{l}\text { center frequency, mean } \\
\text { square frequency, root mean } \\
\text { square frequency }\end{array}$ & 3 \\
\hline $\begin{array}{l}\text { time-frequency } \\
\text { domain (EMD) }\end{array}$ & energy value of each IMF & 8 \\
\hline
\end{tabular}

\section{3 MUlti-SENSOR DATA FUSION}

The multi-dimensional information system provide variously and complementary characteristic information for fault diagnosis. Then, how to fuse information is a vital issue[15]. Multi-sensor data fusion is the data processing in multi levels and multi-dimension to gain the new useful information which is unable to obtain with any single sensor, and then improves the diagnosis accuracy [14].

\section{A. Characteristic feature reduction based on correlation analysis}

After signal processing the feature set includes a large number of characteristic parameters. So many features will lead to complicated structure of classifier, the difficulty of operation, even reduce the diagnosis accuracy. In order to simplify the structure of classifier and improve the model accuracy, the irrelevant or low sensitivity characteristic parameters must be eliminated.

Several characteristic parameters may reflect the same change trend, only in different levels because the influence of measuring point location, sensor types. Firstly these characteristic features will increase the number of classifier input which leads to the complexity of classifier structure. Some researches indicate that the more classifier inputs cannot get better result and high relevant characteristic features may reduce the diagnosis accuracy. So characteristic parameters with high correlation relationship must be removed [17]. All characteristic parameters are processed by fuzzy clustering.

Step 1: matrix of characteristic feature

In order to remove high relevant characteristic features, a number of samples are needed. Please note that these samples must include all kinds of fault type. Then the matrix of characteristic feature $\mathrm{M}$ is got. The data of $\mathrm{M}$ has been normalized.

$$
M=\left[\begin{array}{cccc}
X_{11} & X_{12} & \cdots & X_{1 n} \\
X_{21} & X_{22} & \cdots & X_{2 n} \\
\vdots & \vdots & \ddots & \vdots \\
X_{m 1} & X_{m 2} & \cdots & X_{m n}
\end{array}\right]
$$

Where $\mathrm{n}$ is the number of characteristic value, $\mathrm{m}$ is the number of sample.

Step 2: similar relation matrix

The correlation coefficient of each two columns is calculated which reflects the correlation between the characteristic values. So the similar relation matrix $R_{r \text { is calcu- }}$ lated by the method of correlation coefficient.

$$
\text { The linear correlation coefficient } r_{X_{i j} X_{i J}} \text { is }
$$

$$
r_{X_{i j} X_{i J}}=\frac{\sum_{i=1}^{m}\left(x_{i j}-\bar{x}_{i j}\right)\left(x_{i J}-\bar{x}_{i J}\right)}{\sqrt{\sum_{i=1}^{m}\left(x_{i j}-\bar{x}_{i j}\right)^{2}} \sqrt{\sum_{i=1}^{m}\left(x_{i J}-\bar{x}_{i J}\right)^{2}}}(j, J=1
$$$$
, 2, \ldots, n)
$$

The research shows that clustering by the fuzzy similar matrix can reduce the calculation volume comparing with the fuzzy equivalence matrix. And it is more suitable to deal with high-dimensional data[18].

\section{Step 3: fuzzy clustering}

According to the principle of fuzzy clustering, after setting a certain threshold $\lambda$, the characteristic parameters will then be divided into several classes. The number of class is decided by the value of $\lambda$. The number of sample and characteristic parameter, the type of fault must taken into consideration when the value of $\lambda$ is determining.

The characteristic parameters within the same class have the high correlation. If there is not only one element in the same class, one characteristic parameter can be selected as the input of classifier at random in theory. And the selected methods by some evaluation functions such as revised Euclidean distance or be determined by search 
optimization or genetic algorithms are researched by some experts [19].

\section{B. Primary diagnosis based on single classifier}

There are many different kinds of classifier whose theory and algorithm is different. In field of fault diagnosis Bayesian classifier, neural network classifier and Support Vector Machine (SVM) are used widely. The method and result based on single classifier will introduce in the part of experiment. The result of experiment shows that every kind of classifier is not perfect which can not apply in all conditions. Even for the same type of fault, different classifiers have different diagnosis accuracy due to algorithm. That is to say, each kind of classifier has some limitations and the results of different classifier form the complementary relation. This is the foundation of multiple classifiers fusion.

\section{Final diagnosis based on multiple classifiers fusion}

In the method of multiple classifier fusion it establishes a simulation process of human decision. Each classifier's output is a factor which would be taken into consideration to make the final diagnosis decision-making. The multiple classifier fusion method based on fuzzy comprehensive evaluation is introduced in this paper. The diagnostic accuracy of each classifier is the evaluation parameters to assign weights of classifiers.

The output of each member classifi${ }_{\text {er }} E_{i}=\left\{y_{i 1}, y_{i 2}, \cdots, y_{i m}\right\}_{(\mathrm{i}=1,2, \ldots \mathrm{N}, \mathrm{N} \text { is the number }}$ of classifiers) can be seen as a discrete variable.

Therefore the member classifier's output entropy formula is

$$
H_{y_{j}}=\left\{\begin{array}{c}
-K \sum_{i=1}^{m} y_{i j} \ln y_{i j}, \text { the result is correct } \\
1, \text { the result is not correct }
\end{array}\right.
$$

Where the value of the constant $\mathrm{K}$ is related to the

$$
\begin{aligned}
& \text { number of fault type } \mathrm{m} \text {, take } K=\frac{1}{\ln m} \text {, thus } \\
& 0 \leq H_{y_{j}} \leq 1
\end{aligned}
$$

A certain number of known samples are selected to test for each member classifier. The entropy values of all the outputs of member classifiers are calculated according to the formula (4). In order to eliminate the influence caused by individual factors, $\overline{e_{j}}$ the average entropy of each member classifier is calculated .

The degree of deviation

$$
d_{y_{j}}=1-\bar{H}_{y_{j}}
$$

Each member classifier weight is

$$
\omega_{y_{j}}=\frac{d_{y_{j}}}{\sum_{j=1}^{J} d_{y_{j}}}
$$

The weight vector $A=\left(\omega_{1}, \omega_{2}, \omega_{3}, \ldots \ldots . \omega_{N}\right)$ is derived.

The result of every classifier is fused though the Eq.(10) by the way of fuzzy comprehensive evaluation. B is the final output results. According to the principle of maximum membership, the type of fault is determined and the final diagnosis result is given.

$$
B=A \circ R
$$

There are two advantages of the method of fuzzy comprehensive evaluation.

(1) According to a certain number of prior knowledge, the important level of each classifier in the final decision is determined and the weight value of each classifier is distributed objectively.

(2) The output of fuzzy comprehensive evaluation result is in a vector form which is a fuzzy subset. The result is represented in a variety of fault types of membership which describes the fuzzy nature of fault.

\section{4 EXPERIMENTS}

In order to demonstrate the effectiveness of the method proposed in this paper, experiments are carried out on the part of bearing. According to the location and degree, the bearing wear fault are divided into ten types which includes ball bearing retention's severe and mild wear, inner ring's severe wear and mild wear, outer ring's severe and mild wear, ball's mild, moderate and severe wear and normal. Three acceleration sensors are mounted at measuring point $1,2,3$ to monitor the vibration signals in $\mathrm{X}, \mathrm{Y}$, $\mathrm{Z}$ directions. One noise sensor is mounted at measuring point 4 and one temperature sensor is mounted at measuring point 5 , as shown in fig. 3 .

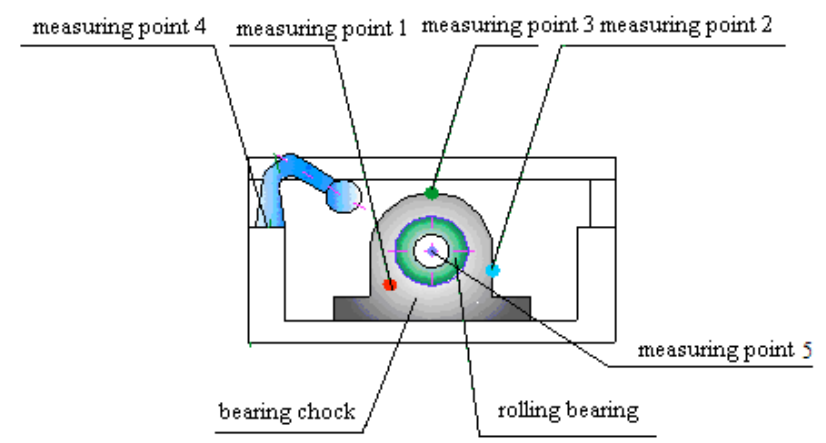

Figure 3. fault specimen of bearing

In the experiment three international parameters of follow error of shaft, the control deviation and the actual speed of encoder are utilized which extracted by machine tool servo system information [21].

According to the method mentioned in section 2, after feature extraction feature vector of each measuring point is formed with 176 characteristic parameters. In order to reduce the amount of calculation, so before the fuzzy clustering, the characteristic features are chosen based on attributes reduction of rough set firstly and 17 characteristic features are remaining which are listed in table I. Then the 17 remained characteristic features data are further analyzed using fuzzy cluster analysis to obtain a fuzzy cluster trend diagram, as shown in fig 4. Ten samples of each kind of fault are selected at random and the 
matrix of characteristic feature $M_{100 \times 17}$ is gained after the normalization of original data matrix. The normalized functions is 'mapminmax $(\mathrm{M}, 0,1)$ '. According to the result of fuzzy cluster trend diagram, 12 classes are finally chosen in the experiment.

The select method of characteristic features in the same class is randomly. The result of characteristic feature reduction is listed in table II .

In order to prove that the characteristic features in the same class have the similar classification ability, the experiment is carried out. The characteristic features in the same class are combined with other categories respectively, and the same BP neural network structure is used to recognize patterns. The result is list in tableIII. Most characteristic value combinations have the better recognition rate, but the training time is different.

The classifiers of BP neural network, RBF network and SVM that are widely applied in fault diagnosis are selected in this paper. Because of different algorithms the advantages of these three classifiers are also different. Firstly three models of each kind of classifier is set up and trained respectively. Please note the output of the BP network and RBF network can be directly used as a vector of the evaluation matrix $R$ after normalization. But the output of SVM is generally the result of classification then the output must be converted into the form of failure's probability value which can be used as a vector of the judgment matrix $R$. All kinds of failure probability values are obtained with the parameters of 'probability_estimates' in the program of SVM. Then 100 groups of sample data are chosen to determine the weight of BP, RBF and SVM according to the way described in part 3.2.2.

The weights of the classifiers are $0.3717,0.2416$ and 0.3867 . In order to validate the proposed method, another 50 groups of sample are chosen to test. The experiment results show that the fault type can be diagnosed correctly if the members of classifier's conclusions are the same. If there is a conclusion conflict between the single classifiers, about $70 \%$ of the sample can identify the fault types after fusion correctly. The correct rate after fusion by fuzzy comprehensive evaluation is higher than any single classifier. This shows the effectiveness of the proposed diagnosis model and proves the method of single classifier integration based on fuzzy comprehensive evaluation is feasible.

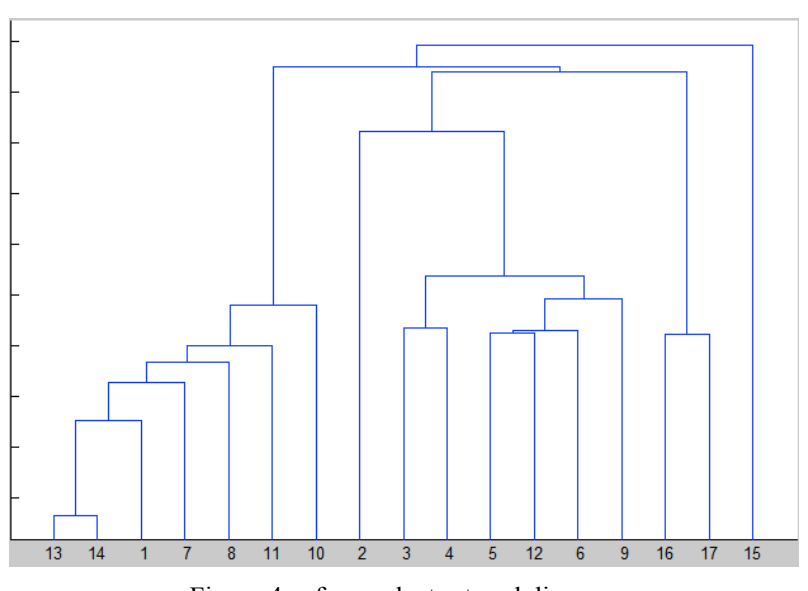

Figure 4. fuzzy cluster trend diagram
TABLE II.

RESULT OF CHARACTERISTIC FEATURE REDUCTION

\begin{tabular}{|l|l|l|}
\hline No. measuring point & $\begin{array}{l}\text { Remained character- } \\
\text { istic feature by } \\
\text { rough set }\end{array}$ & $\begin{array}{l}\text { Remained character- } \\
\text { istic feature after } \\
\text { fuzzy clustering }\end{array}$ \\
\hline measuring point 1 & $\begin{array}{l}\text { E1,E3,E5, skewness, } \\
\text { square frequency, } \\
\text { center frequency }\end{array}$ & $\begin{array}{l}\text { E1,E3,E5,square fre- } \\
\text { quency, center frequen- } \\
\text { cy }\end{array}$ \\
\hline measuring point 2 & $\begin{array}{l}\text { E1,skewness, mean } \\
\text { square frequency }\end{array}$ & E1 \\
\hline measuring point 3 & $\begin{array}{l}\text { E2, center frequency }, \\
\text { average root of fre- } \\
\text { quency }\end{array}$ & $\begin{array}{l}\text { E2,center frequency, } \\
\text { average root of fre- } \\
\text { quency }\end{array}$ \\
\hline measuring point 4 & E2 & E2 \\
\hline measuring point 5 & $\begin{array}{l}\text { waveform factor, } \\
\text { pulse factor }\end{array}$ & pulse factor \\
\hline internal information & $\begin{array}{l}\text { skewness of contour } \\
\text { error, kurtosis factor } \\
\text { of control deviation }\end{array}$ & $\begin{array}{l}\text { kurtosis factor of } \\
\text { control deviation }\end{array}$ \\
\hline
\end{tabular}

TABLE III.

CLASSIFICATION ABILITY OF DIFFERENT CHARACTERISTIC FEATURES IN SAME CLASS(20 SAMPLES)

\begin{tabular}{|c|c|c|c|}
\hline $\begin{array}{c}\text { No. of characteristic fea- } \\
\text { ture }\end{array}$ & $\begin{array}{c}\text { Recognition } \\
\text { rate }\end{array}$ & $\begin{array}{c}\text { training } \\
\text { steps }\end{array}$ & $\begin{array}{c}\text { TRAINING } \\
\text { TIME(s) }\end{array}$ \\
\hline $1,2,3,5,7,8,9,10,11,13,15,16$ & $80 \%$ & 109 & 0.6867 \\
\hline $1,2,3,5,7,8,9,10,11,14,15,16$ & $85 \%$ & 264 & 1.0623 \\
\hline $1,2,3,12,7,8,9,10,11,13,15,16$ & $50 \%$ & 150 & 0.7212 \\
\hline $1,2,3,6,7,8,9,10,11,13,15,16$ & $75 \%$ & 297 & 1.2017 \\
\hline $1,2,3,6,7,8,9,10,11,13,15,17$ & $80 \%$ & 75 & 0.4686 \\
\hline $1,2,4,6,7,8,9,10,11,13,15,17$ & $100 \%$ & 85 & 0.4931 \\
\hline
\end{tabular}

\section{4 CONCLUSIONS}

(1) Multi-source information is the direct source of the original information for $\mathrm{CNC}$ machine tool fault diagnosis. The configuration of the information source must be reasonable because necessary and sufficient information must provide for fault diagnosis. In addition to the traditional external sensor information sources, internal sources of CNC machine tools itself is set in this method. The two types of information sources can form the complementation relation effectively, so as to reduce or eliminate the uncertainty in diagnosis process caused by incomplete data. The experiments also show that the characteristic values from the internal information source have an important influence to the diagnosis decision.

(2) The essence of information fusion is in the process of multi-level and multi-dimensional fusion to find new information. There are data layer, feature layer and decision-making three different levels fusions in the diagnosis model. The signals from different types and different sample frequencies are aligned in time and space in the first data layer fusion. The characteristic values are compressed by the feature layer fusion, provide sensitive characteristic features for diagnosis. Compressed sensitive feature set is seen as a diagnostic network input. Level of each classifier in the diagnosis of parallel network respectively establishes a preliminary conclusion of the test sample, which is the equivalent result of a number of experts. Based on fuzzy comprehensive evaluation of secondary diagnosis network, the value is distributed objectively to the experts for comprehensive evaluation. The process of diagnosis is simulating human thinking and methods, integration in the process of diagnosis level 
of diversification, and organic combination of data fusion and fusion model, which are able to reduce or avoid the failure phenomenon of missed diagnosis and misdiagnosis.

(3) Diagnosis model, signal processing, feature extraction and diagnosis decision-making, etc., all are taken certain measures to reduce or eliminate the uncertain factors of diagnosis process, so as to improve the diagnostic accuracy.

\section{REFERENCES}

[1] Alberto José Álvares, João Carlos Espíndola Ferreira. Web turning: Teleoperation of a CNC turning center through the Internet [J]. Journal of Materials Processing Technology, 2006,179(1):251259 http://dx.doi.org/10.1016/j.jmatprotec.2006.03.096

[2] Http://www.lgmazak.com.cn/

[3] YE Z, Sadeghian A, Wu B. Mechanical fault diagnostics for induction motor with variable speed drives using adaptive Neurofuzzy inference system [J]. Electric Power systems research, 2006,76(9):742-752

[4] Sampath Suresh, Singh Riti. An integrated fault diagnostics model using genetic algorithm and neural networks [J]. ASME Transactions on Journal of Engineering for Gas Turbines and Power,2006,128:49-56

[5] Samanta B, Nataraj C. Use of particle swarm optimization for machinery fault detection [J]. Engineering Applications of Artificial intelligence, 2009,22:308-316 http://dx.doi.org/10.1016/ j.engappai.2008.07.006

[6] LEI Ya-guo, HE Zheng-jia. Advances in applications of hybrid intelligent fault diagnosis and prognosis technique $[\mathrm{J}]$. Journal of vibration and shock, 2011,30(9):129-135

[7] Mohammad K J, Langari R. A hybrid intelligent system for fault detection and sensor fusion [J]. Applied Soft Computing, 2009,9:415-422 http://dx.doi.org/10.1016/j.asoc.2008.05.001

[8] HOU Zhao-wen, ZHANG Zhou-suo. Hybrid intelligent fault diagnosis based on granular computing [C]. 2009 IEEE International Conference on Granular Computing, Nanchang, China: 219224 ,

[9] LIANG Steven y, HECKER R L , LANDERS R G. Machining Process Monitoring and Control: The State-of-the-art[J]. Journal of Manufacturing Science and Engineering, 2004, 5(12):297-310

[10] VOLKER Plapper, MANFRED Weck. Sensorless Machine Tool Condition Monitoring Based on Open NCs[C] // IEEE Power Engineering Society, International Conference on Robotics and Automation, 2001: 3104-3108

[11] ZHOU Yu-qing, MEI Xue-song, JIANG Ge-dong, et al., Technology on large scale numerical Control machine tool condition monitoring based on built-in sensors [J], Ji Xie Gong Cheng XueBao, 2009(4): 125-130

[12] STONE G C. Advancements during the past quarter century in online monitoring of motor and generator winding insulation [C]. IEEE Transactions on Dielectries and Electrical Insulation, UK. 2002:1057 1058
[13] HUANG N E, SHEN Z, LONG S R, et al.. The empirical mode decomposition and the Hilbert spectrum for nonlinear and nonstationary time series analysis $[\mathrm{J}]$. Proceedings of the Royal Society of London, 1998,454(1):903-995

[14] DONG Wei-hua, LI Xian'en, LIN Xiang-guo, et al.. A Bidimensional Empirical Mode Decomposition Method for Fusion of Multispectral and Panchromatic Remote Sensing Images. RemoteSens.2014,6,8446-8448 http://dx.doi.org/10.3390/rs6098446

[15] LUDMILA I, KUNCHEVA. Fuzzy versus nonfuzzy in combining classifiers designed by boosting [J]. IEEE Transactions on Fuzzy Systems, 2003, 11 (6): 729-741 http://dx.doi.org/10.1109/TFUZZ. 2003.819842

[16] ZHAO Zhi-hong, YANG Shao-pu, SHEN Yong-jun. Machinery fault feature extraction based on independent component analysis and correlation coefficient $[\mathrm{J}]$, Journal of Vibration and Shock, 2013(6): 67-72

[17] YUAN Jin-sha, SHANG Hai-kun, Wang Yu, et al.. Pattern recognition for partial discharge based on correlation coefficient matrix and probabilistic neural networks, Power System Protection and Control, 2013(13):110-115

[18] LIU Qi,LIN Huai-zhong,CHEN Chun. Application of the Maximum Tree Algorithm for Fuzzy Clustering in the Clustering of Web Page [J]. Application Research of Computers,2004,21(11)

[19] YUAN Wei-qi, JING Lan-tao, Lin Sen, et al.. Hand-shape feature selection method based on class distinctive degree and correlation [J], Chinese Journal of Scientific Instrument, 2013(8): 1787-1794

[20] YANG Lun-biao, GAO Ying-yi. Principium of fuzzy mathematics and its application (5th Edition)[M]. South China University of Technology Press, Guangzhou, 2011:100-104

[21] SONG Ping. CNC table feed system fault diagnosis [D], Qingdao: Qingdao Technological University, 2013:42-63

\section{AUTHORS}

WEN Yan is with School of Mechanical Engineering, Qingdao Technological University, Qingdao, CO266520, China.

TAN Ji-wen is with School of Mechanical Engineering, Qingdao Technological University, Qingdao, CO266520, China.

ZHAN Hong is with School of Mechanical Engineering, Qingdao Technological University, Qingdao, CO266520, China.

SUN Xian-bin is with School of Mechanical Engineering, Qingdao Technological University, Qingdao, CO266520, China.

This work was supported in part by .The Research Fund for the Doctoral Program of Higher Education (No. 20123721110001), Projects of Colleges and Universities Science and Technology Plan of ShanDong Province (J13LB11) and Basic Research Projects of QingDao City Science and Technology Plan (No. 12-1-4-4-(3)-JCH). Submitted 17 September 2015. Published as resubmitted by the authors 23 January 2016. 